\title{
The prevalence of metabolic syndrome in people with severe mental illness: a mediation analysis
}

\author{
Giuseppe Carrà̀ ${ }^{1}$, Francesco Bartoli ${ }^{2}$, Daniele Carretta ${ }^{2}$, Cristina Crocamo ${ }^{3}$, Alberto \\ Bozzetti $^{2}$, Massimo Clerici ${ }^{2}$, Paul E. Bebbington ${ }^{1}$
}

Word count: Abstract 207; Text 3,689; Tables: 4

${ }^{1}$ Mental Health Sciences Unit, Faculty of Brain Sciences, University College London. London, UK. ${ }^{2}$ Department of Surgery and Interdisciplinary Medicine, University of Milano Bicocca, Milano, Italy.

${ }^{3}$ Department of Mental Health, San Gerardo Hospital, Monza, Italy.

Address for correspondence:

Francesco Bartoli, MD, PhD, University of Milano-Bicocca, Department of Surgery and Interdisciplinary Medicine, Via Cadore 48, 20900 Monza (MB), Italy. Tel. +390392333982; Fax +390392332277 E-mail: f.bartoli@ campus.unimib.it 


\section{ABSTRACT}

Background People with severe mental illness (SMI) generally have high rates of metabolic syndrome (MetS).

Proposed explanations remain conjectural. Relatively little is known about Mets in SMI in Southern Europe, an area with generally healthy dietary traditions.

Purpose To establish prevalence rates of MetS in an Italian sample, testing hypotheses about putative reasons for the excess in the SMI group.

Methods We compared the prevalence and correlates of MetS in inpatients with SMI and controls randomly chosen from patients undergoing routine maxillofacial surgery. We employed formal tests of mediation.

Results The MetS prevalence rate was $26.1 \%$ in the SMI group and $15.9 \%$ in the comparison group. After controlling for age, people with SMI were three times more likely to have MetS than their non-SMI counterparts. Smoking and a family history of cardiovascular disease were strongly related to MetS in both groups. However, these factors could not explain the excess of MetS in the SMI group, and we found no effect of antipsychotic dose.

Conclusions SMI remained prominent in increasing the likelihood of MetS in this low prevalence population, and putative determinants of MetS were common to people with SMI and to controls. Explanations for high MetS rates in SMI may lie in health behaviours other than smoking.

Keywords: Mental Disorders; Metabolic syndrome; Comorbidity; Prevalence; Risk factors. 


\section{INTRODUCTION}

People suffering from severe mental illness (SMI) have a shorter life expectancy than the general population [1]. Suicide and accidental injury account for $30-40 \%$ of this excess mortality [2], while cardiovascular disease (CVD) causes the vast majority of deaths from physical illness [3]. The increased frequency of CVDs in SMI is likely to involve a number of risk factors commonly considered modifiable in the general population. These include smoking, poor diet, lack of exercise, excessive alcohol intake, obesity, diabetes, and dyslipidaemia [4]. The cluster of abnormal clinical and metabolic findings defining the metabolic syndrome (MetS) is predictive of CVDs [5], and may be of particular importance in SMI. The most frequently used definition of MetS is that set out by the Adult Treatment Panel ATP-III of the US National Cholesterol Education Program (NCEP), [6].

The large Clinical Antipsychotic Trials of Intervention Effectiveness (CATIE) study applied the NCEP definition of MetS to people with chronic schizophrenia. The odds of having MetS were two and three times greater respectively in men and women with schizophrenia than the age-adjusted prevalence rate of $23.7 \%$ in American adults [7]. However, the prevalence rates of MetS in people with SMI actually vary strikingly in different geographic locations, ranging from $3.9 \%$ to $54 \%$ [8]. Some of this variation may result from sampling biases in relation to sociodemographic and clinical characteristics. Nevertheless, local social characteristics and dietary habits may also have an important bearing. Understanding the inconsistencies in the prevalence of MetS in people with SMI should reveal the impact of local social circumstances, and whether variations are just as apparent as in the general population. Moreover, good prevalence data are crucial in terms of the financial resources to be allocated, and the training needed for planning basic primary prevention and routine diagnostic screening for MetS and CVD risk factors in this vulnerable group. Finally, the variation may have aetiological implications.

Three key candidates for explaining the association are predisposition due to genetic overlap between SMI and MetS, antipsychotic medication, and detrimental health behaviours. Certainly, a genetic association between SMI and MetS cannot be ruled out [9]. Antipsychotic medication has been associated with an increased prevalence of MetS: there is a dose effect for CVD risk factors and some agents seem to have an especially adverse impact [10]. The fact that over a third of people with established schizophrenia taking antipsychotic medication can, by the age of 38 , be identified biochemically to be at high risk of diabetes emphasises the importance of prevention [11]. Finally, people with SMI are particularly likely to smoke, to have a poor diet, and to avoid exercise, and this may be linked to deficiencies in relevant skills, self-efficacy and motivation [12]. Each of these aetiological possibilities may be illuminated by studying them in geographical contexts with particular attributes. 
The population of countries in Southern Europe usually show reduced CVD-related mortality rates, possibly because of healthy Mediterranean dietary habits [13]. This region thus offers opportunities for studying the prevalence of MetS in relation to family history, to antipsychotic medication and to adverse health behaviours in people with SMI. If these are important causes of MetS, the relative odds of MetS in people with SMI should be relatively increased in the context of healthy eating habits at the population level. However, previous epidemiological studies in Southern Europe have been hampered by methodological issues. Samples have often been based on clinical convenience [14], and control groups have been unsuitable for a number of reasons, lacking relevant healthy comparison groups [15], using comparison samples from different populations [16] or based on large existing administrative databases [17].

\section{Aims of the study}

The present study was designed to examine the prevalence of MetS in a representative cohort of people with SMI in Italy. We hypothesised, i) that, after adjusting for relevant clinical and social variables, people with SMI would have higher MetS rates than those free of mental disorder, and ii) that the association between SMI and MetS would be mediated by a family history of CVD, the level of antipsychotic medication, and smoking habits.

\section{MATERIALS AND METHODS}

\section{Setting and study design}

The study took place in Monza-Brianza (MB), a mixed urban and rural province in Northern Italy, which includes districts ranging from the most to the least deprived in Italy. It was approved by the local research ethics committee (code "Non solo mente").

The study was a cross-sectional comparison of people with and without SMI, recruited from patients admitted to San Gerardo University Hospital (MB). This eleven hundred bed general hospital has a comprehensive range of medical and surgical inpatient services, and a 20 bed ward for acute mental health care serving a catchment area of 319,000 inhabitants.

\section{Sample}


All patients with a DSM-IV diagnosis of SMI admitted to the Mental Health Inpatient Unit between March 2011 and February 2012 were approached for informed, signed, consent to the study. If they agreed, they were interviewed on the day before their planned discharge in order to check their diagnosis. SMI was defined as at least one 12-month DSMIV/SCID diagnosis of schizophrenia, schizophreniform disorder, schizoaffective disorder, delusional disorder, brief psychotic disorder, shared psychotic disorder, psychotic disorder NOS, and bipolar I-II disorders, together with a GAF score of less than 60 for at least one month in the past year [18]. In the same period, all inpatients from the maxillofacial surgery unit admitted for planned, routine (non-emergency) surgical treatment were approached in order to establish an eligible list for the general hospital comparison group. We chose this clinical population as unlikely to suffer more from chronic physical disorders than the general population. To be eligible, people from both groups had to be at least 18 years old and living in a private residence. People were excluded from the comparison group if they had received a formal SMI diagnosis, had ever taken an antipsychotic medication, or were on the caseload of a mental health service.

\section{Procedure}

Fully trained consultant psychiatrists administered the 12-month patient version of the SCID-I [Structured Clinical Interview for DSM-IV] [19] to identify SMI diagnoses within the index group.

\section{Definition of the metabolic syndrome}

MetS was defined by the presence of three or more of the following criteria [12]:

- abdominal obesity (waist circumference $>102 \mathrm{~cm}$ in men and $>88 \mathrm{~cm}$ in women)

- fasting triglycerides concentration $\geq 150 \mathrm{mg} / \mathrm{dl}$

- $\quad$ systolic/diastolic blood pressure $\geq 130 / 85 \mathrm{mmHg}$ (or on antihypertensive medication)

- high density lipoprotein (HDL) cholesterol $<40 \mathrm{mg} / \mathrm{dl}$ in men and $<50 \mathrm{mg} / \mathrm{dl}$ in women

- fasting glucose concentration $\geq 110 \mathrm{mg} / \mathrm{dl}$ (or on insulin or hypoglycaemic medication).

Where it was infeasible to measure waist circumference (i.e., for a small proportion of bedridden patients) we substituted an abdominal obesity criterion equivalent to a body mass index (BMI) value $\geq 28.8 \mathrm{~kg} / \mathrm{m} 2$, without losing reliability [20].

\section{Data collection}

General socio-demographic information (age, gender, education, employment status, family situation) was collected. We elicited clinical data covering current medication for hypertension, dyslipidaemia and diabetes, clinical and family 
history of CVDs (e.g., myocardial infarction, acute heart failure, acute coronary syndrome, and stroke), and selfreported smoking habits. Participants with SMI were asked about current antipsychotic medication, and the information cross-checked in clinical records. The amounts prescribed in the past six months were converted to average chlorpromazine equivalent doses [21-24]. The routine admission procedure established height and weight (on electronic scales, with the minimal necessary clothing), and BMI. Waist circumference was measured according to standard guidelines [25]. Resting blood pressure was established at the beginning and end of the interview, and the mean value was determined. Fasting plasma levels of glucose, triglycerides and total cholesterol (LDL plus HDL) were measured.

\section{Statistical analysis}

Our sample size calculation was based on an Italian national prevalence for MetS of 20\% [26] and meta-analytic data suggesting MetS rates of $40 \%$ in people with SMI [27]. Groups of 119 participants would allow the demonstration of a 20\% differences in MetS rates, given 90\% power and a two sided 5\% level of significance, as previously reported [7]. In total, 208 and 196 individuals were eligible for the SMI and comparison groups respectively. We used simple random sampling to obtain the requisite number of subjects from the enrolled populations. The sample size calculation was also appropriate for the chosen tests for mediation, even though these have less statistical power.

Analyses were carried out using Stata for Windows version 10.0 SE. We used logistic regression in a structured approach to analyse the effect of SMI on the prevalence of MetS. We controlled for age and sex, and then added the variables we had identified as putative explanations for the increased rate of MetS in people with SMI, using SobelGoodman tests for mediation as framed in the sgmediation command in Stata. We used mediation analyses to test whether the relationship between SMI (independent variable) and MetS (dependent variable) is direct or whether putative mediator variables account for the relationship between them. Our data allowed us to consider three candidate mediators. The first possibility is that there is an overlap between the genes associated with MetS and SMI. We used a family history (FH) of CVD as a partial proxy for the genetic basis of MetS. If there is genetic overlap, this would be expressed as mediation of the SMI-MetS link by FH CVD. The second possibility is that treatment with antipsychotic drugs increases the risk of MetS. If so, there should be a dosage effect, and antipsychotic dose should fulfil the requirements for mediation. Finally, the explanation might lie in harmful health behaviours, and we therefore examined the contribution of smoking status.

Having ascertained that: 1) there is a direct effect between SMI and MetS; and 2) the putative mediator variables are associated with MetS, we used Sobel-Goodman tests for mediation to assess if the association between SMI and MetS is reduced by a significant amount (partial mediation), or is completely accounted for, by the mediator (total mediation). Each putative mediator was entered in separate models in order to investigate their individual impact on the 
relationship. If the entry of the mediator was accompanied by a statistically significant effect on the dependent variable, together with a reduction of the SMI effect, partial mediation can be deduced (indirect effect). On the other hand, if the effect of the mediator lacks statistical significance, all that can be inferred is a direct effect of SMI on MetS.

\section{RESULTS}

No eligible patient refused to participate in the study. In table 1, people with SMI are compared with the control participants. The prevalence rate for ATP III metabolic syndrome in people with SMI was $26.1 \%, 60 \%$ higher than in the comparison group $(15.9 \%)$. The latter is almost identical to that found $(16.2 \%)$ in a large epidemiological study of the same geographical area [28]. This supports the representativeness of our control group, and, indirectly, our prevalence estimates for the SMI group. People in the SMI group were less often married or cohabiting, and fewer were employed. There was no difference in educational level or age. However, there was an unexpected under-representation of males in the SMI group, approaching conventional levels of statistical significance $(\mathrm{p}=0.07)$, and the excess prevalence of MetS in the SMI group was restricted to female participants. Rates in the SMI group were higher in females than in males, while the reverse was true in the control group.

\section{Table 1 about here}

Because of these sex differences, we analysed formally whether sex moderated the relationship between SMI and the metabolic syndrome (Table 2). While SMI and sex were both significantly related to MetS, an interaction term linking sex with SMI was not. However, controlling for sex strengthened the relationship between SMI and MetS, and the degree of statistical significance increased.

\section{Table 2 about here}

We next carried out logistic analysis of the effect of SMI on the prevalence of MetS (Table 3), controlling for sociodemographic variables significantly related in univariate analysis. SMI, age, smoking status and a family history of CVD remained significantly associated with MetS, while family situation and gender did not.

\section{Table 3 about here}

We then tested our specific hypotheses relating to potential mediators of the relationship between SMI and MetS, that is, a family history of cardiovascular disease, antipsychotic medication dose and smoking status. These analyses each included age as an independent variable because of its established effect on MetS. The results are presented in table 4. As we have seen, a family history of CVD was strongly associated with the metabolic syndrome. However, the 
proportion of the effect of SMI on MetS that was mediated by family history was minuscule: virtually all the effect was therefore direct. Thus, in our data, a family history of CVD cannot explain the excess of MetS in people with SMI. What about the effect of medication dosage? It should be noted that in the SMI group, 115 of 119 (97\%) were taking antipsychotics (atypical in 110). The median chlorpromazine dose was moderate, at $242 \mathrm{mg}$ daily (interquartile range of $150-483 \mathrm{mg}$ ). The indirect component of the SMI-MetS link due to dose of medication appears at first sight to be appreciable, representing about $32 \%$ of the total effect. However, the direction of effect was the reverse of what we hypothesised - it seems as if the link between SMI and MetS was attenuated by increasing doses of antipsychotic medication. It should be noted that the contribution of dosage to the model was non-significant $(\mathrm{p}=0.16)$. Finally we analysed the effect of smoking status. In this instance about $10 \%$ of the overall link between SMI and MetS was indirect, through smoking. However, this effect was, once more, non-significant ( $\mathrm{p}=0.19)$ : in our sample, smoking is also unable to account for the relationship between SMI and MetS.

\section{Table 4 about here}

\section{DISCUSSION}

We compared an epidemiologically representative random sample of inpatients with SMI with an appropriate comparison group. DSM-IV research diagnoses for SMI were based on formal diagnostic interviews. The role of possible confounders was addressed at the stage of design for the index and the comparison groups (through random sampling) and at the stage of analysis for the whole sample (by adjusting for a selected set of variables).

\section{Main findings}

The prevalence of $26 \%$ for MetS rates in our SMI group is consistent with earlier studies from other countries belonging to the low cardiovascular risk region of southern Europe [29]. Similar rates have been reported in outpatients samples from Spain [17], although a prevalence of ATP III MetS of 34.2\% in a Turkish cohort was higher $(27.7 \%$ for men, $39.3 \%$ for women) [16]. Values of $32.5 \%$ were reported from the United States in a recent meta-analysis, and more significantly, our results are lower than any so far reported from inpatient samples, as for this subgroup the pooled rate of MetS was 30.4\% [8]. The only comparable rates (24\%) come from a Spanish cross-sectional study in patients diagnosed with schizophrenia admitted to short-stay hospitalisation units [15].

The age-adjusted general population prevalence of MetS is similar in the USA [30] and in Italy [26]. The single largest US study so far [7] used a large general population comparison sample [30], and reported an unadjusted OR of suffering 
from Mets, given SMI, of $1.94(95 \% \mathrm{CIs}=1.52-2.47)$. The analogous unadjusted OR from our data was $1.85(95 \% \mathrm{CIs}=$ 0.98-3.50). Thus, given SMI, the relative odds of having MetS seem roughly equivalent in the USA and in Italy.

However, after adjustment for age and sex, people suffering from SMI were three times more likely to suffer from MetS than our chosen controls. Thus, in this low prevalence population with traditionally healthy dietary habits, SMI does have a prominent effect in increasing the likelihood of MetS. It is also clear, from our analysis as a whole, that a family history of CVD and smoking are MetS risk factors in both the SMI case group and our control sample. A history of cardiovascular disease increased fivefold the likelihood of suffering from MetS: this is consistent with data from the general population [5]. The corresponding odds ratio for smoking was around 2.5, somewhat greater than in other reports from the Italian general population $(\mathrm{OR}=1.34)$ [31].

We investigated whether these factors, along with the dose of antipsychotic medication, could account for the excess of MetS in the SMI group, by using formal tests of mediation. Smoking can be taken here as an example of adverse health behaviour, and we used a family history of CVD as a partial proxy for genetic overlap between MetS and SMI. In the event, none of these factors could explain the higher rates of MetS in the SMI group.

It has been argued that people with SMI have a particular susceptibility to metabolic abnormalities and there may be genetic risk factors common to diabetes and schizophrenia [32]. However, over 100 genes are associated with schizophrenia, the associations are very weak (OR <1.15), and between them they explain only $5 \%$ of the variance [33]. In order to account for much of the SMI-MetS link, a large number of these genes would also have to be associated with MetS. This seems unlikely, to say the least, and so it is not surprising that family history of CVD did not mediate the SMI-MetS link.

Our mediation analysis yielded a central value implying that $10 \%$ of the link between SMI and MetS might be mediated by smoking. However this effect was not statistically significant. A role for smoking does remain possible because we were unable to collect detailed information about smoking levels, and its association with MetS is likely to be dosedependent [34]. Poor health behaviours as a whole may yet lie at the heart of the high prevalence of MetS in people with SMI.

While age and familial predisposition are non-modifiable risk factors, it has been strongly argued that poor diet and poor health habits are proper targets for intervention. People with SMI are relatively ignorant of CVD risk factors and interventions and appear to give less priority to their physical health needs [12]. Their motivation to change their health behaviour may be affected by disbelief, both that such change can reduce risks and that they have the ability to change, though their motivation for weight loss may be just as strong as in the general population [35]. Modifying and 
implementing programmes to change the health beliefs and behaviours of the severely mentally ill population clearly remains a challenge.

In our age-controlled mediation analysis, we were unable to find any association between the dose of antipsychotic medication and the likelihood of suffering from MetS. This is surprising: if antipsychotic medication tends to induce MetS in the people who take it, it should account for some of the excess of MetS in people with SMI, and there ought to be a dose effect. The absence of mediation by antipsychotic dose may suggest that, at the population level at least, such drugs do not contribute much to the excess, or perhaps that much of the medication effect is already apparent at low dosage. We quantified the use of antipsychotic agents in the previous six months, but MetS may require a longer period to become apparent, and thus reflect cumulative dosage levels [36]. Equally, we could not assess intermittent antipsychotic usage, often related to poor compliance. Finally physicians may seek preferentially to lower medication dosage in people with increasing BMI. People with SMI may have a propensity to develop metabolic abnormalities regardless of antipsychotic medication [37], but our failure to find a dose effect must be set against evidence that adverse cardio-metabolic risks appear within weeks of commencing anti-psychotics, particularly weight gain, glucose dysregulation and hypercholesterolemia [38], not to mention sudden cardiac death [39]. Thus we should certainly opt for the least metabolically impairing antipsychotics.

\section{Limitations}

The setting, the manner of delivery of interventions and the exclusion criteria are relatively similar to those found elsewhere, allowing reasonable generalizability to similar populations in Western countries. Nevertheless, as MetS was assessed in treated populations, our findings may differ from those in people out of contact with services. Although the procedure for identifying SMI was based on the use of a standardised instrument (the SCID-I), the reliability of ratings was not formally established during the study, and additional clinical characteristics could be assessed in order to control their confounding role. Furthermore, sample size calculations were based on a $40 \%$ event rate in people with SMI according to the International literature. However this is much higher than that observed in our local sample (26\%), possibly affecting power and bringing risk of random error. In addition, we could study only smoking but the role of other adverse health behaviours (e.g., poor diet and lack of exercise) deserves further research in people with SMI. While attempts were made to ensure comparable recruitment procedures, acute-phase treatment might have exacerbated the metabolic disturbances. Furthermore, although people from the maxillofacial surgery unit admitted for planned, routine (non-emergency) surgical treatment have been chosen because they are likely to be free from any medical chronic condition, they cannot be perfect proxies for the general population. Cross-sectional studies constrain causal inference, but the fact that there was a strong association of MetS with smoking but not with the dose of 
antipsychotic medication makes a differential causal effect plausible, particularly as in both cases there is a putative mechanism.

\section{CONCLUSION}

Despite the comparatively low prevalence of MetS in this study, it still has significant public health implications for the marginalised group of people with SMI, whose disadvantages often include further comorbid issues such as substance dependence and cognitive impairment [40, 41] and a wide range of severe physical illnesses [10]. The Mediterranean countries have traditionally shown lower cardiovascular mortality rates than other developed countries, so there is a particular need for monitoring, diagnosing, treating, and educating people with SMI about the associated metabolic abnormalities. In particular, health promotion for people with SMI should aim to raise awareness of modifiable health behaviours such as smoking, overeating, consumption of relatively unhealthy foods and inactivity [42, 43]. Future research should include not only the follow-up of samples in different regions in order to assess the relative contribution of life style and health behaviours to MetS, but also appropriate guidelines [12] about effective health promotion interventions targeting physical activity, eating habits and smoking cessation. 


\section{Acknowledgements}

We thank Peter Tyrer (Centre for Mental Health at Imperial College London) for the comments on an earlier version of the article. Our thanks to all the patients and staff from the Mental Health Inpatient Unit and the Maxillofacial Surgery Unit, San Gerardo University Hospital (MB-I).

\section{Conflict of interest}

The authors declare that they have no conflict of interest. 


\section{REFERENCES}

[1] Saha S, Chant D, McGrath J (2007) A systematic review of mortality in schizophrenia: is the differential mortality gap worsening over time? Arch Gen Psychiatry 64:1123-1131.

[2] Tiihonen J, Lonnqvist J, Wahlbeck K, Klaukka T, Niskanen L, Tanskanen A et al (2009) 11-year follow-up of mortality in patients with schizophrenia: a population based cohort study (FIN11 study). Lancet 374:620-627.

[3] Lahti M, Tiihonen J, Wildgust H, Beary M, Hodgson R, Kajantie E et al (2012) Cardiovascular morbidity, mortality and pharmacotherapy in patients with schizophrenia. Psychol Med 42:2275-2285.

[4] Yusuf S, Hawken S, Ounpuu S, Dans T, Avezum A, Lanas F et al (2004) Effect of potentially modifiable risk factors associated with myocardial infarction in 52 countries (the INTERHEART study): case-control study. Lancet 364:937-952.

[5] Galassi A, Reynolds K, He J (2006) Metabolic syndrome and risk of cardiovascular disease: a meta-analysis. Am J Med 119:812-819.

[6] Expert Panel on Detection, Evaluation, and Treatment of High Blood Cholesterol in Adults (2001) Executive Summary of The Third Report of The National Cholesterol Education Program (NCEP) Expert Panel on Detection, Evaluation, And Treatment of High Blood Cholesterol In Adults (Adult Treatment Panel III). JAMA 285:2486-2497.

[7] McEvoy JP, Meyer JM, Goff DC, Nasrallah HA, Davis SM, Sullivan L et al (2005) Prevalence of the metabolic syndrome in patients with schizophrenia: baseline results from the Clinical Antipsychotic Trials of Intervention Effectiveness (CATIE) schizophrenia trial and comparison with national estimates from NHANES III. Schizophr Res 80:19-32.

[8] Mitchell AJ, Vancampfort D, Sweers K, Van Winkel R, Yu W, De Hert M (2013) Prevalence of Metabolic Syndrome and Metabolic Abnormalities in Schizophrenia and Related Disorders-A Systematic Review and Meta-Analysis. Schizophr Bull 39:306-318.

[9] Mulder H, Cohen D, Scheffer H, Gispen-de Wied C, Arends J, Wilmink FW et al (2009) HTR2C gene polymorphisms and the metabolic syndrome in patients with schizophrenia: a replication study. $\mathrm{J}$ Clin Psychopharmacol 29:16-20. 
[10] De Hert M, Correll CU, Bobes J, Cetkovich-Bakmas M, Cohen D, Asai I et al (2011) Physical illness in patients with severe mental disorders. I. Prevalence, impact of medications and disparities in health care. World Psychiatry 10:52-77.

[11] Manu P, Correll CU, Van Winkel R, Wampers M, De Hert M (2012) Prediabetes in patients treated with antipsychotic drugs. J Clin Psychiatry 73:460-466.

[12] Buhagiar K, Parsonage L, Osborn DP (2011) Physical health behaviours and health locus of control in people with schizophrenia-spectrum disorder and bipolar disorder: a cross-sectional comparative study with people with non-psychotic mental illness. BMC Psychiatry 11:104.

[13] Kokkinos P, Panagiotakos DB, Polychronopoulos E (2005) Dietary influences on blood pressure: the effect of the Mediterranean diet on the prevalence of hypertension. J Clin Hypertens 7:165-70.

[14] Salvi V, Albert U, Chiarle A, Soreca I, Bogetto F, Maina G (2008) Metabolic syndrome in Italian patients with bipolar disorder. Gen Hosp Psychiatry 30:318-323.

[15] Bernardo M, Cañas F, Banegas JR, Casademont J, Riesgo Y, Varela C; RICAVA Study Group (2009) Prevalence and awareness of cardiovascular risk factors in patients with schizophrenia: a cross-sectional study in a low cardiovascular disease risk geographical area. Eur Psychiatry 24:431-441.

[16] Yazici MK, Anil Yağcioğlu AE, Ertuğrul A, Eni N, Karahan S, Karaağaoğlu E et al (2011) The prevalence and clinical correlates of metabolic syndrome in patients with schizophrenia: findings from a cohort in Turkey. Eur Arch Psychiatry Clin Neurosci 261:69-78.

[17] Sicras-Mainar A, Blanca-Tamayo M, Rejas-Gutiérrez J (2008) Metabolic syndrome in outpatients receiving antipsychotic therapy in routine clinical practice: a cross-sectional assessment of a primary health care database. Eur Psychiatry 23:100-108.

[18] Carrà G, Sciarini P, Segagni-Lusignani G, Clerici M, Montomoli C, Kessler RC (2011) Do they actually work across borders? Evaluation of two measures of psychological distress as screening instruments in a non AngloSaxon country. Eur Psychiatry 26:122-127.

[19] Spitzer RL, Williams JB, Gibbon M, First MB (1992) The Structured Clinical Interview for DSM-III-R (SCID). I: History, rationale, and description. Arch Gen Psychiatry 49:624-629. 
[20] Rejas J, Bobes J, Arango C, Aranda P, Carmena R, Garcia-Garcia M (2008) Concordance of standard and modified NCEP ATP III criteria for identification of metabolic syndrome in outpatients with schizophrenia treated with antipsychotics: A corollary from the CLAMORS study. Schizophr Res 99:23-28.

[21] Atkins M, Burgess A, Bottomley C, Riccio M (1997) Chlorpromazine equivalents: a consensus of opinion for both clinical and research applications. Psychiatr Bull 21:224-226.

[22] Lehman AF, Steinwachs DM (1998) Translating research into practice: the Schizophrenia Patient Outcomes Research Team (PORT) treatment recommendations. Schizophr Bull 24:1-10.

[23] Woods SW (2003) Chlorpromazine equivalent doses for the newer atypical antipsychotics. J Clin Psychiatry 64:663-667.

[24] Kroken RA, Johnsen E, Ruud T, Wentzel-Larsen T, Jørgensen HA (2009) Treatment of schizophrenia with antipsychotics in Norwegian emergency wards, a cross-sectional national study. BMC Psychiatry 9:24.

[25] World Health Organization [WHO] (2011) Waist circumference and waist-hip ratio: report of a WHO expert consultation. Geneva, 8-11 December 2008. Geneva, Switzerland. http://whqlibdoc.who.int/publications/2011/9789241501491_eng.pdf. Accessed 12 August 2013.

[26] Miccoli R, Bianchi C, Odoguardi L, Penno G, Caricato F, Giovannitti MG et al (2005) Prevalence of the metabolic syndrome among Italian adults according to ATP III definition. Nutr Metab Cardiovasc Dis 15:250254.

[27] Osborn DPJ, Wright CA, Levy G, King MB, Deo R, Nazareth I (2008) Relative risk of diabetes, dyslipidaemia, hypertension and the metabolic syndrome in people with severe mental illnesses: Systematic review and metaanalysis. BMC Psychiatry 8:84.

[28] Mancia G, Bombelli M, Corrao G, Facchetti R, Madotto F, Giannattasio C et al (2007) Metabolic syndrome in the Pressioni Arteriose Monitorate E Loro Associazioni (PAMELA) study: daily life blood pressure, cardiac damage, and prognosis. Hypertension 49:40-47.

[29] Graham I, Atar D, Borch-Johnsen K, Boysen G, Burell G, Cifkova R et al (2007) Fourth Joint Task Force of the European Society of Cardiology and Other Societies on Cardiovascular Disease Prevention in Clinical Practice. European guidelines on cardiovascular disease prevention in clinical practice: executive summary. Eur Heart J 28:2375-2414. 
[30] Ford ES, Giles WH, Dietz WH (2002) Prevalence of the metabolic syndrome among US adults: findings from the third National Health and Nutrition Examination Survey. JAMA 287:356-359.

[31] Masulli M, Riccardi G, Galasso R, Vaccaro O (2006) Relationship between smoking habits and the features of the metabolic syndrome in a non-diabetic population. Nutr Metab Cardiovasc Dis 16:364-370.

[32] Fernandez-Egea E, Bernardo M, Donner T, Conget I, Parellada E, Justicia A et al (2009) Metabolic profile of antipsychotic-naive individuals with non-affective psychosis. Br J Psychiatry 194:434-438.

[33] Ripke S, O'Dushlaine C, Chambert K, Moran JL, Kähler AK, Akterin S et al (2013) Genome-wide association analysis identifies 13 new risk loci for schizophrenia. Nat Genet 45:1150-1159.

[34] Chen CC, Li TC, Chang PC, Liu CS, Lin WY, Wu MT et al (2008) Association among cigarette smoking, metabolic syndrome, and its individual components: the metabolic syndrome study in Taiwan. Metabolism 57:544-548.

[35] Parks J, Radke AQ (2008) Obesity reduction and prevention strategies for individuals with serious mental illness. Alexandria: National Association of State Mental Health Program Directors (NASMHPD) Medical Directors Council.

[36] American Diabetes Association (2004) Consensus development conference on antipsychotic drugs and obesity and diabetes. Diabetes Care 27:596-601.

[37] Nielsen J, Skadhede S, Correll CU (2010) Antipsychotics associated with the development of type 2 diabetes in antipsychotic-naïve schizophrenia patients. Neuropsychopharmacology 35:1997-2004.

[38] Foley DL, Morley KI (2011) Systematic review of early cardiometabolic outcomes of the first treated episode of psychosis. Arch Gen Psychiatry 68:609-616.

[39] Ray WA, Chung CP, Murray KT, Hall K, Stein CM (2009) Atypical antipsychotic drugs and the risk of sudden cardiac death. N Engl J Med 360:225-235.

[40] Carrà G, Johnson S (2009) Variations in rates of comorbid substance use in psychosis between geographical areas and mental health settings in the UK. Soc Psychiatry Psychiatr Epidemiol 44:429-447. 
[41] Carrà G, Johnson S, Bebbington P, Angermeyer MC, Heider D, Brugha T et al (2012) The lifetime and pastyear prevalence of dual diagnosis in people with schizophrenia across Europe: findings from the European Schizophrenia Cohort (EuroSC). Eur Arch Psychiatry Clin Neurosci 262:607-616.

[42] Bartoli F, Carrà G, Crocamo C, Carretta D, Clerici M (2013) Bipolar Disorder, Schizophrenia and Metabolic Syndrome. Am J Psychiatry 170:927-928.

[43] Bartoli F, Carrà G, Crocamo C, Carretta D, Clerici M (2013) Metabolic Syndrome in People Suffering from Posttraumatic Stress Disorder: A Systematic Review and Meta-Analysis. Metab Syndr Relat Disord 11:301308. 
Table 1 Socio-demographic characteristics, metabolic syndrome and severe mental illness (SMI)

\begin{tabular}{|l|c|c|c|}
\hline Variable & With SMI (n=119) & Without SMI (n=119) & $P$ \\
\hline Age: Mean (SD), yrs & $47.2(14.8)$ & $49.4(19.7)$ & 0.32 \\
\hline Sex: Male & $51(42.9 \%)$ & $65(54.6 \%)$ & 0.069 \\
\hline Education: $\geq$ High school & $58(48.7 \%)$ & $56(47.1 \%)$ & 0.80 \\
\hline Married/cohabiting: & $28(23.5 \%)$ & $64(54.7 \%)$ & $<0.001$ \\
\hline Employed: & $58(48.7 \%)$ & $104(87.4 \%)$ & $<0.001$ \\
\hline ATP III Metabolic Syndrome & $31(26.1 \%)$ & $19(15.9 \%)$ & 0.056 \\
Males & $12(23.5 \%)$ & $13(20 \%)$ & \\
Females & $19(27.9 \%)$ & $6(11.1 \%)$ & \\
\hline
\end{tabular}


Table 2 Severe mental illness, sex and the metabolic syndrome: logistic regression

\begin{tabular}{|l|c|c|c|}
\hline Variables & Odds Ratio & 95\% CIs & P \\
\hline SMI & 1.85 & $0.98-3.51$ & 0.058 \\
\hline SMI (males) & 1.23 & $0.51-2.99$ & 0.65 \\
SMI (females) & 3.10 & $1.14-8.44$ & 0.027 \\
\hline SMI & 4.53 & $1.54-13.36$ & 0.006 \\
Sex (reference female) & 3.07 & $1.00-9.43$ & 0.05 \\
SMI*sex & 0.41 & $0.10-1.69$ & 0.215 \\
Age & 1.06 & $1.03-1.08$ & 0.0001 \\
\hline
\end{tabular}


Table 3 Variables associated with ATP III Metabolic Syndrome: logistic regression

\begin{tabular}{|c|c|c|c|}
\hline Variables & Odds Ratio & $95 \% \mathrm{CI}$ & $\boldsymbol{P}$ \\
\hline SMI & 3.42 & 1.49 to 7.86 & 0.004 \\
\hline Age & 1.03 & 1.01 to 1.07 & 0.005 \\
\hline Sex & 0.77 & 0.36 to 1.63 & 0.499 \\
\hline Ever smoked & 2.48 & 1.09 to 5.61 & 0.029 \\
\hline Family situation $^{a}$ & 2.17 & 0.96 to 4.88 & 0.062 \\
\hline Family history of cardiovascular disease & 5.10 & 1.88 to 13.83 & 0.001 \\
\hline
\end{tabular}

${ }^{\text {a}}$ Reference category: living alone 
Table 4 Tests of mediation between SMI and MetS: Family history of cardiovascular disease (FH CVD), Antipsychotic medication and Smoking status

\begin{tabular}{|l|c|c|c|c|}
\hline \multicolumn{1}{|c|}{ Term } & Coefficient & Standard error & $\mathbf{t}$ & $\boldsymbol{P}$ \\
\hline FH CVD & .008 & .053 & 0.15 & 0.89 \\
\hline SMI & .12 & .051 & 2.29 & 0.023 \\
\hline Age & .007 & .001 & 4.51 & 0.0001 \\
\hline Sobel-Goodman test of mediation & & & $\mathbf{Z}$ & \\
\hline Indirect effect & -.0001 & .0008 & -0.12 & 0.90 \\
\hline Direct effect & .116 & .051 & 2.29 & 0.022 \\
\hline Total effect & .116 & .051 & 2.29 & 0.022 \\
\hline \multicolumn{1}{|c|}{ Term } & Coefficient & Standard error & $\mathbf{t}$ & $\boldsymbol{P}$ \\
\hline CPZ equivalent dose & -.0002 & .0001 & -1.44 & 0.15 \\
\hline SMI & .165 & .058 & 2.07 & 0.04 \\
\hline Age & .007 & .001 & 4.60 & 0.0001 \\
\hline Sobel-Goodman test of mediation & & & $\mathbf{Z}$ & \\
\hline Indirect effect & -.040 & .028 & -1.42 & 0.16 \\
\hline Direct effect & .165 & .058 & 2.84 & 0.004 \\
\hline Total effect & .125 & .051 & 2.45 & 0.014 \\
\hline \multicolumn{1}{|c|}{ Term } & Coefficient & Standard error & $\mathbf{t}$ & $\boldsymbol{P}$ \\
\hline Smoker vs. non-smoker & .098 & .051 & 1.91 & 0.06 \\
\hline SMI & .105 & .051 & 2.07 & 0.04 \\
\hline Age & .007 & .001 & 4.76 & 0.0001 \\
\hline Sobel-Goodman test of mediation & & & $\mathbf{Z}$ & \\
\hline Indirect effect & .011 & .009 & 1.31 & 0.19 \\
\hline Direct effect & .105 & .051 & 2.07 & 0.04 \\
\hline Total effect & .116 & .051 & 2.29 & 0.022 \\
\hline
\end{tabular}

